\title{
Les innovations démocratiques dans un contexte de fusions municipales : le cas de Gatineau
}

\author{
Guy Chiasson \\ Université du Québec en Outaouais
}

\section{Introduction}

C e texte discute de l'impact de la réforme municipale de 2000 sur la démocratie locale en se penchant plus spécifiquement sur le cas de Gatineau. Bien que les fusions municipales ne signifient pas en soi un transfert de pouvoir du centre (Québec) vers la périphérie (les localités et les régions) et qu'elles ne fassent ainsi pas toujours partie de ce qu'on entend par la décentralisation, on pourrait facilement montrer que la politique de fusion s'inscrit dans une volonté de Québec de consolider le pouvoir local en vue de la

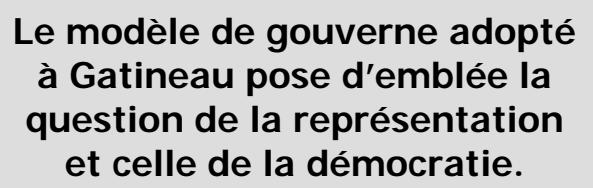

qui sera confirmé par la suite par les élus locaux. Le modèle de gouverne adopté à Gatineau pose d'emblée la question de la représentation et celle de la démocratie. Cette question sera d'ailleurs au cœur d'un important débat qui se tiendra autour d'un exercice de planification stratégique pour la nouvelle ville de Gatineau. Ce débat a amené la Ville à s’engager à mettre en place une "gouvernance participative » en instaurant un certain nombre de mécanismes allant en ce sens. Il est intéressant de noter que, comme à Montréal et Québec, ce modèle de gouverne a été soumis, en 2003, au test des défusions. Les référendums tenus en juin 2004 dans les anciennes villes de décentralisation éventuelle de nouvelles responsabilités. Je prendrai donc pour acquis que les réformes municipales font partie d'une amorce de décentralisation du pouvoir entre Québec et les villes. Dans quelle mesure la réorganisation de la gouvernance des agglomérations urbaines facilite-telle le renouvellement des pratiques démocratiques locales ? Plus particulièrement, je vais me pencher sur le cas que je connais le mieux, celui de Gatineau, pour voir comment la création de la nouvelle Ville est propice à l'émergence de formes de démocratie où le pouvoir local est mieux partagé entre les élus et la société civile.

On se souviendra que le gouvernement québécois a imposé, par l'entremise de la loi 170, la fusion de plusieurs municipalités dans les agglomérations urbaines de Montréal, Québec et de l'Outaouais. Le modèle de fusion qui sera adopté dans l'Outaouais avec la création de Gatineau sera passablement différent du modèle à trois paliers (arrondissement, ville et communauté métropolitaine) mis en vigueur à Montréal et Québec. À Gatineau, le gouvernement retiendra l'option d'un seul palier de gouverne locale, choix
Masson-Angers, Buckingham, Aylmer et Hull laisseront tout de même intacte la ville de Gatineau, tout en lui épargnant la mise en place d'un conseil d’agglomération.

\section{L'oubli de la question démocratique}

Poser la question de la démocratie participative a de quoi surprendre ceux qui ont suivi de près la réforme municipale de 2000 au Québec. La plupart des analystes ont été très durs envers cette réforme, lui reprochant tour à tour son manque d'innovation par rapport aux politiques précédentes ou son incapacité à s'attaquer sérieusement aux problématiques centrales (la question du financement ou celle de l'étalement urbain, par exemple) du monde municipal québécois. Jean-Pierre Collin fera justement remarquer l'absence, dans la politique de fusions québécoises, de deux éléments qu'il juge centraux dans un processus de réforme du secteur public local : une révision du partage des pouvoirs entre niveaux de gouvernement et ensuite «l'ouverture des institutions locales et supra locales à la participation de la société civile à la décision 
et à la gestion relatives aux services publics locaux en favorisant une démocratie participative ${ }^{1}$. Cette interprétation se trouve confirmée par une lecture des documents publics entourant la réforme. Le Livre blanc $^{2}$, par exemple, fait très peu référence à la démocratie, laissant ainsi largement le terrain aux opposants qui s'en sont servis allègrement! Lorsqu'il le fait, c'est pour faire valoir que, dans le contexte de petites municipalités, la démocratie perd tout son sens à cause de la faiblesse des institutions locales. Ces propos nous rappellent que la réforme reste solidement accrochée au registre classique de la démocratie représentative, sans s'aventurer sur la question de la participation des citoyens dans les nouvelles villes consolidées.

Le projet de décentralisation n'était donc pas accompagné d'une volonté ou encore d'outils permettant de faciliter l'émergence de formes de démocratie participative dans les nouvelles villes fusionnées. En cela, la politique municipale québécoise diffère de l'expérience d'autres États, notamment la France, qui ont rendu obligatoire la mise en place de structures participatives dans les villes les plus populeuses.

L'absence de la démocratie participative dans le discours de la politique de fusion n'a cependant pas empêché l'émergence (inattendue ?) d'innovation démocratique dans les villes issues de la fusion. C'est donc dire que les acteurs locaux ont fait une place à l'innovation démocratique dans la construction des nouvelles villes et cela, malgré les orientations proposées par le projet de décentralisation imaginé par Québec.

\section{La création politique de la nouvelle Ville de Gatineau}

Les analystes des questions urbaines au Canada ont souvent fait remarquer l'inféodation des municipalités par rapport aux provinces. Selon l'expression consacrée, les municipalités sont des "créatures des provinces ». Cette position de faiblesse est toujours très actuelle, surtout sur le plan légal. Le cas de Toronto a montré comment la fusion s'est faite sans aucune considération de la volonté des acteurs locaux (élus autant que citoyens). Malgré cette responsabilité ultime qu'ont les provinces de définir le contour des institutions municipales, ce sont les acteurs locaux et les dynamiques locales qui donnent vie à ces institutions et qui viennent en quelque sorte les modeler.
Aussi, on peut difficilement comprendre l'impact des fusions municipales sans tenir compte des dynamiques locales à l’œuvre.

\section{Le projet de décentralisation n'était donc pas accompagné d'une volonté ou encore d'outils permettant de faciliter l'émergence de formes de démocratie participative dans les nouvelles villes fusionnées.}

\section{Une dynamique particulière : les rapports transfrontaliers}

À Gatineau, la question complexe du rapport avec Ottawa est sans aucun doute une dynamique dont il faut tenir compte pour bien comprendre le modèle de fusion préconisé en Outaouais. L’Outaouais urbain fait partie, à bien des égards, d'une même agglomération urbaine avec Ottawa et est depuis longtemps influencée par la force d'attraction d'Ottawa. Le gouvernement du Québec et certains acteurs locaux ont souvent cherché à combattre ce rapprochement transfrontalier en marquant leur territoire par l'entremise d'institutions proprement québécoises : la Communauté régionale de l'Outaouais, la Société d'aménagement de l'Outaouais, la Communauté urbaine de l'Outaouais. La création de la ville de Gatineau s'inscrit dans cette lignée. Le gouvernement ontarien avait fait la fusion de la ville d'Ottawa un an plus tôt et on se devait de faire contrepoids du coté québécois. C’est pourquoi, à mon avis, on a retenu un modèle à un seul palier, comme celui mis en place à Ottawa, plutôt que de suivre le modèle plus complexe de Montréal et Québec.

La fusion imposée par Québec mettait nécessairement en branle un processus de construction de la nouvelle ville à partir des cinq anciennes municipalités. Cela nécessitait d'importants réajustements administratifs (harmonisation des conventions collectives, définition des contours de l'appareil administratif). C'est le Comité de transition, selon un mandat confié par le gouvernement, qui s'est occupé de ces tâches complexes ${ }^{3}$. Ce qui est intéressant cependant, c'est que le processus de construction de la nouvelle ville ne s'est pas arrêté là. La construction de la ville s'est poursuivie sur le terrain politique. C'est donc dire que les élus de Gatineau ont profité de la fusion pour impulser un 
modèle de ville qui allait bien au-delà des opérations administratives d'harmonisation. Cette opportunité aura permis de soulever un certain nombre de questions, dont celle du rapport avec Ottawa et celle de la participation de la société civile dans le processus décisionnel municipal. Ces deux questions seront en fait fortement imbriquées l'une dans l'autre puisque la participation de la société civile dans les affaires municipales sera souvent vue comme une ressource indispensable pour s'assurer que la ville puisse tirer son épingle du jeu des dynamiques transfrontalières ${ }^{4}$.

Je me contenterai ici de présenter quelques initiatives qui constituent des indices de la présence d'un ferment de démocratie participative à Gatineau.

\section{Un exercice de planification stratégique}

À la suite de l'élection du premier conseil municipal de la nouvelle ville, une large démarche de planification stratégique a été lancée. Cet exercice, qui visait à identifier les priorités pour la nouvelle ville sur un horizon de 25 ans, a fait appel à plusieurs reprises à la mobilisation et à l'expertise des citoyens. Tout d'abord, cet exercice de planification a été animé par une commission, la Commission des choix stratégiques, composée d'élus (3) dont le maire, de certains fonctionnaires clés (2) et d'une majorité de représentants de la société civile provenant de divers secteurs d'activités (6). Les travaux de cette Commission se sont appuyés largement sur le dynamisme de la société civile locale, que ce soit par l'entremise de dépôts de mémoires, de séances de consultation auprès d'organismes, de sondages, etc. D'ailleurs, une des priorités parmi les quatre grandes identifiées par la planification était d'arriver à une " gouvernance participative ». Cette expérience me semble significative dans la mesure où elle équivaut à une concession des élus qui acceptent que la définition des priorités urbaines ne soit pas uniquement de leur ressort.

\section{Les élus de Gatineau ont profité de la fusion pour impulser un modèle de ville qui allait bien au-delà des opérations administratives d'harmonisation.}

En continuité avec l'exercice de planification stratégique, la Ville a commencé à se doter de politiques qui vont servir à définir les orientations municipales dans plusieurs secteurs d'activités. Deux politiques, la Po- litique culturelle et la Politique familiale, ont été adoptées jusqu'ici. On retiendra de ces politiques qu'elles ont été élaborées par des Commissions municipales : la Commission des arts, des lettres, de la culture et du patrimoine ainsi que le Comité de la famille. Ces institutions, à l'image de la Commission des choix stratégiques, réunissent des élus, des fonctionnaires et des citoyens tout en faisant une large place à ces derniers. Les politiques stipulent que ce sont ces Commissions qui auront dans l'avenir le mandat de gérer la politique et de la revoir, le cas échéant.

\section{Démocratie et positionnement métropolitain}

Ces initiatives peuvent être interprétées comme une volonté d'institutionnaliser la participation des citoyens tant au plan de la définition des grandes priorités de la Ville qu'au plan de ses orientations sectorielles. Par rapport à la gestion municipale antérieure, dominée par une démocratie représentative à l'échelle des municipalités et un pouvoir des experts à l'échelle de l'ancienne Communauté urbaine, l'expérience de la Ville de Gatineau représente une innovation démocratique qui se rapproche plus de la délibération. Certes le pouvoir des élus et celui des experts n'ont pas disparu et ne sont pas en voie de l'être, mais il semble qu'ils soient maintenant amenés à faire plus de place à d'autres acteurs, dont les citoyens.

Il y a lieu de parler d'un renforcement de la démocratie, même s’il n'est pas irréversible. Cet effort de démocratisation doit être mis en rapport avec la position particulière de Gatineau dans l'agglomération urbaine de la Capitale nationale. Les mesures mises de l'avant s'inscrivent assez clairement dans un projet qui est celui de donner une identité à la nouvelle ville. L'identité gatinoise est fragile à deux niveaux. D'une part, il faut de toute évidence assurer une identification des citoyens à la nouvelle ville plutôt qu'aux anciennes communautés municipales. D'autre part, la ville doit aussi trouver sa place et sa légitimité face à Ottawa. C'est-à-dire qu'elle est amenée à se positionner en même temps sur deux tableaux : celui de la région de la capitale nationale et celui de capitale régionale de l'Outaouais. Un regard rapide sur les documents de la planification stratégique permet de voir assez facilement que le fond identitaire de Gatineau ne va pas de soi dans le contexte de sa double appartenance. Cette préoccupation identitaire vient souvent en tête de liste des défis que devra relever la 
nouvelle ville. Le plan stratégique ${ }^{5}$ constate, par exemple, que les défis en matière de développement économique et social seront relevés " en affirmant notre identité propre et distincte comme ville francophone, enrichie de sa diversité culturelle, au sein de la grande agglomération urbaine de la région de la capitale nationale; en affirmant notre présence économique dans la grande région urbaine en faisant valoir nos compétences et nos talents particuliers ».

Ce projet ambitieux, qui est celui de construire une nouvelle ville dynamique et forte capable de tirer profit de sa position frontalière exceptionnelle (et dont la planification stratégique est l'une des manifestations concrètes), ne pourra cependant pas se réaliser sans un engagement soutenu des forces vives de la société civile. "Sur le plan économique, nous devons saisir les occasions qui s'offrent à nous de nous diversifier, en exploitant par exemple les secteurs de pointe ou qui font appel à notre spécificité culturelle. Du point de vue social, notre participation individuelle est nécessaire pour améliorer la situation. Chacun peut, par exemple, appuyer à la mesure de ses moyens ces organismes du milieu pleins de bonne volonté qui ont besoin de notre soutien. Le développement de notre ville commence par chacun d'entre nous, individuellement ou collectivement, en collaboration avec nos administrations publiques, notre milieu des affaires et nos organismes communautaires et sociaux. Notre réussite dépendra de notre habileté à travailler et à trouver ensemble des solutions. Le rôle de notre administration municipale pourrait, dans ce cas-ci, consister à coordonner ces efforts et mettre en commun ces potentiels ${ }^{6}$.

Le lien entre l'ouverture démocratique et le projet de construction de la ville est très important pour notre propos dans la mesure où il vient colorer le type de démocratie et en même temps son rapport avec la solidarité. Dans un contexte où le projet est celui de construire une nouvelle ville, les acteurs prioritaires sont ceux qui disposent en quelque sorte de ressources utiles pour mener à bien ce projet. Que ce soit de façon consciente ou pas de la part des organisateurs, les travaux de la Commission des choix stratégiques ont réussi à rejoindre assez facilement certains types d'acteurs sociaux peu impliqués auparavant, mais qui se retrouvaient bien dans les nouvelles orientations proposées par le plan stratégique (groupes préoccupés par le développement durable, par la culture et le pa- trimoine, mais également des organismes régionaux). D’autres groupes ont semblé beaucoup moins présents. On peut se demander si la planification stratégique a surtout fait place à des acteurs représentant des groupes bien organisés qui avaient ultimement quelque chose (de l'expertise ou d'autres ressources complémentaires à celles de l'appareil municipal) à offrir au processus. Les acteurs moins bien pourvus ont été moins présents, même si certains groupes associés au développement des quartiers défavorisés l'ont été. Dans le cadre de la planification stratégique, peu de choses ont été faites pour s'assurer que les personnes et les groupes plus défavorisés aient eux aussi une participation significative. Les multiples invitations lancées aux citoyens n'ont pas été suffisantes pour assurer une telle représentativité.

\section{Par rapport à la gestion municipale antérieure, dominée par une démocratie représentative à l'échelle des municipalités et un pouvoir des experts à l'échelle de l'ancienne Communauté urbaine, l'expérience de la Ville de Gatineau représente une innovation démocratique qui se rapproche plus de la délibération.}

\section{Conclusion}

L'objectif de départ de ce texte était d'évaluer dans quelle mesure la politique de fusion, considérée comme un premier pas vers la décentralisation, permet d'instaurer une démocratie participative. Nous avons pu voir que la fusion, couplée avec une préoccupation importante d'assurer une meilleure place pour Gatineau dans l'agglomération qu'elle partage avec Ottawa, a amené la Ville à mettre en place divers mécanismes faisant appel à l'expertise et à la participation des citoyens. Est-ce qu'on doit voir ces initiatives comme des manifestations d'une démocratie participative à Gatineau ? Certains travaux de recherche récents portant sur les expériences de démocratie à l'échelle des métropoles occidentales mettent sérieusement en garde contre la tentation de voir ces initiatives comme faisant partie d'un projet de démocratisation. Très souvent, ces initiatives ne feraient que confirmer ou cautionner les pouvoirs des élus, semant un doute profond sur le potentiel d'innovation démocratique que recèlent la décentralisation et les restructurations urbaines. Il me semble tout de même que les initiatives 
qui ont pris place à Gatineau sont significatives dans le sens où elles accordent une place réelle aux citoyens dans la définition des orientations politiques de la ville. Cette démocratie participative, il faut s'en rappeler, n'a pas la prétention de remplacer les structures de représentation, mais plutôt de leur servir de prolongement et parfois de contrepoids.

\section{Notes et références}

1 Collin, J.-P. (2002), « La réforme de l’organisation du secteur municipal au Québec: la fin ou le début d'un cycle ? », Organisations et territoires, vol. 11, n 3 , p. 5-13.
2 Ministère des Affaires municipales et de la Métropole (2000). La réorganisation municipale. Changer les façons de faire pour mieux servir les citoyens, Livre blanc sur la réforme municipale, Québec, MAMM, 35 p.

3 Voir Collin, J.-P. et J. Léveillée (2002), « Les comités de transition vers les nouvelles villes d'agglomération : résultats d'une enquête », Organisations et territoires, vol. 11, $n^{\circ} 3$, automne.

4 Voir notamment Ville de Gatineau (2003), Plan stratégique 2003-2007.

5 Ibid.

${ }^{6} \quad$ Ibid. 


\section{Publicité}

Revue PME 